\title{
Soleal and gastrocnemius vein thrombosis in a patient with a muscular hematoma
}

\author{
Asunción María Gonzálvez ${ }^{*}$, Paula Chacón ${ }^{2}$, Florentino García-Sánchez ${ }^{3}$, David Jiménez ${ }^{4}$, Meritxell Mellado ${ }^{5}$ and Manuel Monreal ${ }^{6}$ \\ ${ }^{1}$ Servicio de Medicina Interna, Hospital General de Villalba, Collado Villalba, Madrid, Spain \\ ${ }^{2}$ Servicio de Medicina Interna-Urgencias, Hospital Infanta Sofía, San Sebastián de los Reyes, Madrid, Spain \\ ${ }^{3}$ Servicio de Medicina Interna, Hospital Universitario Príncipe de Asturias, Alcalá de Henares, Madrid, Spain \\ ${ }^{4}$ Servicio de Neumología, Hospital Universitario Ramón y Cajal, Madrid, Spain \\ ${ }^{5}$ Servicio de Angiología y Cirugía Vascular, Hospital del Mar, Barcelona, Spain \\ ${ }^{6}$ Servicio de Medicina Interna, Hospital Universitari Germans Trias i Pujol, Badalona, Barcelona, Spain
}

\begin{abstract}
Background: Isolated soleal and/or gastrocnemius deep vein thrombosis (ISGVT) may be incidentally diagnosed in patients evaluated for other clinical manifestations such as intramuscular hematoma. Thrombosis limited to soleal and gastrocnemius veins is associated with a clinically significant rate of subsequent vein thromboembolism (VTE) development and some authors believe that therapeutic anticoagulation may reduce the incidence of VTE complications.

Case report: A 63-year-old woman presented to the Emergency Room (ER) with left limb pain. She had had a left metatarsal operation the week before and discharged with painkillers and low molecular weight heparin (LMWH) $40 \mathrm{mg}$ daily. A compression ultrasound (CUS) in the ER showed a left thigh hematoma (5 $\times 21 \times 48 \mathrm{~mm}$ ) together with soleal and gastrocnemius deep vein thrombosis.

Conclusions: No treatment guidelines are available for this variant of deep vein thrombosis (DVT) and data regarding the risk factors and natural history of ISGVT are limited. Meticulous ultrasonographic assessment and skillful sonographers may increase ISGVT diagnosis, thus allowing the design of well powered studies that might improve our ISGVT behavior understanding.
\end{abstract}

\begin{abstract}
Abbreviations: CUS: Compression Ultrasound; DVT: Deep Vein Thrombosis; ER: Emergency Room; IDDVT: Isolated Distal Deep Vein Thrombosis; ISGDVT: Isolated Soleal and/or Gastrocnemius Deep Vein Thrombosis; LMWH: Low Molecular Weight Heparin; PE: Pulmonary Embolism; PTS: Post-Thrombotic Syndrome; VTE: Vein Thromboembolism
\end{abstract}

\section{Introduction}

Although isolated soleal and/or gastrocnemius deep vein thrombosis (ISGVT) is diagnosed with increasingly frequency, its clinical relevance and optimal treatment are still unknown. Many studies have focused on tibial and peroneal vein (calf veins) thrombosis [1-3], but the natural history and optimal therapy of ISGDVT are still unknown.

Anatomical considerations should be taken into account. According to the nomenclature of veins of the lower limb [4], soleal and gastrocnemius veins are included in the deep venous system (Figure 1). Thus, it is accepted that DVT and ISGDVT have similar risk factors. The real incidence of ISGDVT is not well known, but it is suspected to be of significance. In an analysis of patients with suspected DVT, thrombus was isolated to the gastrocnemius vein in $17 \%$ of limbs, and the soleal in $20 \%$ [5]. Another study suggested that the soleal and/or grastrocnemial veins may be involved in up to $79 \%$ of limbs found to have thrombosis and $25 \%$ of all leg thromboses may be limited to these vessels [6].

The outcome of patients with untreated ISGDVT is unclear. Its rate of propagation to proximal deep veins and pulmonary embolism (PE) is clinically important in patients with clinically important in patients with DVT, but few, but few prospective studies have addressed this issue in patients with ISGDVT. Interesting information can be derived from studies investigating complications in patients with isolated calf DVT. A blind prospective study showed that proximal extension of isolated distal deep vein thrombosis (IDDVT) occurred within 5-7 days in around $3 \%$ of patients, and over $90 \%$ had complete resolution without anticoagulant treatment [1]. Another recent study concluded that PE was present in $36 \%$ of patients with proximal DVT and in only $13 \%$ of patients with calf DVT, the majority of the latter being minor emboli [7]. Regarding the issue of late sequelae of IDDVT, in an early study on post-thrombotic syndrome (PTS) after acute DVT, it was found that subjects with IDDVT had a PTS score significantly lower than those with proximal DVT [8].

Hence, since ISGDVT is a minor form of presentation of calf vein thrombosis, it could be considered that the rate of complications

Correspondence to: Asunción María Gonzálvez, Servicio de Medicina Interna. Hospital General de Villalba. Collado Villalba, Madrid, Spain, Tel: + 34 638003869; E-mail: asunggasch@gmail.com

Key words: Soleal and/or gastrocnemius vein thrombosis, distal vein thrombosis, diagnosis, treatment

Received: January 03, 2016; Accepted: January 28, 2016; Published: February 02,2016 


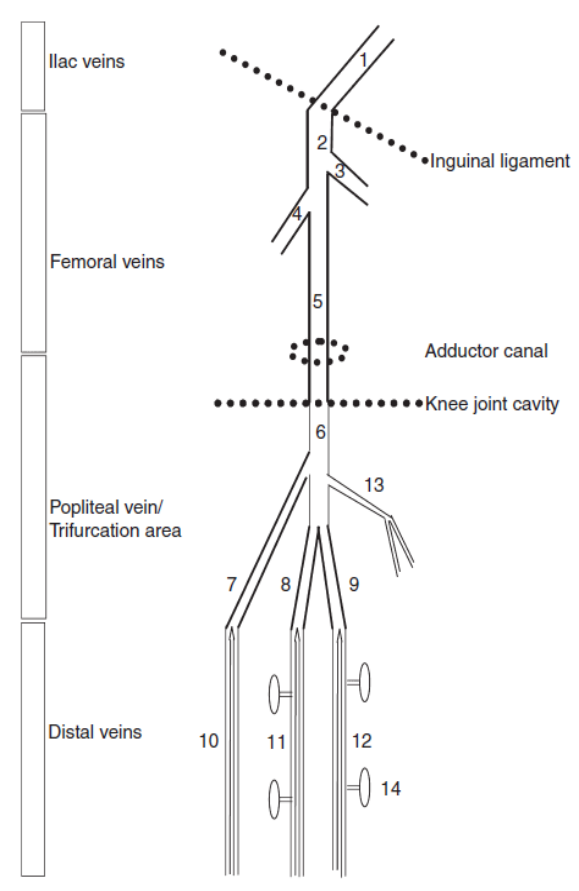

Figure 1. Schematic representation of leg veins as discussed in this review: 1, external iliac vein; 2, common femoral vein; 3 , greater saphenous vein; 4, profound femoral vein; 5 , (superficial) femoral vein; 6, popliteal vein; 7, anterior tibial confluent segment; 8 , posterior tibial confluent segment; 9 , peroneal confluent segment; 10, anterior tibial veins; 11, posterior tibial veins; 12 , peroneal veinss; 13, gastrocnemius muscle veinss(medial head); 14, ssoleuss muscle veins.

derived from DVT limited to soleal and gastrocnemius veins would be similar or even lower than that related to IDDVT. Regarding diagnosis, validation studies have shown that whereas a venous compression ultrasound (CUS) has high accuracy for proximal DVT (thrombosis of the popliteal and more proximal veins) it lacks sensitivity and specificity for distal DVT, even in symptomatic patients [9]. However, it is nowadays accepted that ultrasonographic assessment is the firstline imaging procedure for diagnosis of ISGDVT. The management for patients with ISGDVT is now evolving. Treatment guidelines for ISGDVT do not exist, mostly due to the lack of information on the clinical significance of this entity.

\section{Case report}

A 63-year-old woman was admitted to the ER Department following a six-day history of left limb pain. Her past medical history revealed hypertension and chronic ischemic cardiomyopathy treated with angioplasty and stent 8 years before. She was on Lisinopril and Aspirin treatment.

She had had her left metatarsal operated the week before, with no post-operative complications. She was discharged with painkillers and subcutaneous low molecular weight heparin (LMWH) $40 \mathrm{mg}$ daily. Some days later she presented in the ER with tenderness in her left thigh, where an ischemia sleeve was placed for surgery the week before. On physical examination no signs of inflammation such as erythema or warmth were seen, hence she was discharged with more painkillers. Three days later she referred more pain despite the treatment given. Again, the physical examination revealed no swelling, edema or changes in the left limb circumference.
A CUS was performed in the ER and showed a left thigh hematoma $(5 \times 21 \times 48 \mathrm{~mm})$ together with soleal and gastrocnemius deep vein thrombosis. Thus, there was some concern about which would be the optimal management for this patient.

\section{Discussion}

Soleal and gastrocnemius DVT are diagnosed with increasingly frequency. It has been generally accepted that DVT and ISGDVT share the same risk factors, but there is growing evidence that ISGDVT is particularly associated with transient factors such as hospitalization and recent surgery or trauma, like the present case. Thrombosis in soleal and gastrocnemius veins can occur in the setting of hematomas affecting limb muscles. Recent travel and presence of leg varicosities are also more prevalent in patients presenting with ISGDVT [6], although no varicosities were seen on physical examination and the patient did not refer a recent travel.

Whenever ISGDVT is suspected, the entire deep venous system (between the common femoral vein and popliteal vein distally through the peroneal, anterior tibial, posterior tibial, soleus, and gastrocnemius veins) should be evaluated for compressibility at $1 \mathrm{~cm}$ intervals. Results are considered positive for DVT if a venous segment is not fully compressible. There is no information whether the entire deep venous system was examined in the present case or not.

The detection of ISGDVT with ultrasonographic assessment depends on the clinical pretest probability and on the availability of trained sonographers. Meticulous examination strategy using a previously stablished protocol is likely to avoid inter and intra observer variability as well as to improve sensibility and specificity of the CUS.

As for ISGDVT management, some authors recommend anticoagulation therapy for patients with ISGDVT in order to avoid proximal extension and even PE, however the natural history of ISGDVT is unknown. Data regarding this issue are limited and come from very few small studies with conflicting findings. Schwarz et al. [10] prospectively followed two cohorts of consecutive patients with ISGDVT, the first receiving a therapeutic dose of LMWH for 10 days plus compression therapy, the second receiving compression therapy only. Progression to the calf veins occurred in $13 / 32$ patients in the cohort without anticoagulation and in only one out of 52 patients under LMWH therapy. No patient in either arm of the study was diagnosed with PE. In contrast, another recent study reported no differences in 3 months progression rates between patients with ISGDVT receiving 10 days of therapeutic LMWH and compression therapy or compression therapy only [11]. This discrepancy may be explained by the fact that in the first study more patients presented persistent risk factors such as cancer or prolonged immobilization.

MacDonald et al. [12] reported a trivial rate of propagation to the popliteal vein and no cases of PE or thigh DVT in a prospective analysis on 135 limbs managed without anticoagulation and monitored with serial duplex examination for three months. This study showed an overall $16.3 \%$ rate of DVT, including calf $(13.3 \%)$ and popliteal DVT (3\%) but no proximal iliae, common femoral or superficial femoral DVT or PE. Furthermore, $90.9 \%$ of all DVT occurred within a 2-week time period. On the other hand, Gillet et al. [13] prospectively followed 128 patients with ISGDVT with therapeutic anticoagulation and showed that anticoagulation therapy protected against VTE events, and that extension or recurrence occurred frequently after cessation of therapy. The authors found that $18.8 \%$ of patients had at least one episode of VTE recurrence, including a $4.7 \%$ rate of subsequent PE. 
Reflecting the lack of information on this topic, the most recent consensus from the American College of Chest Physicians regarding antithrombotic therapy for VTE provides no treatment guidelines specific for patients with ISGDVT [14]. Some authors suggested that anticoagulation for ISGDVT may be unnecessary, while others have recommended anticoagulation therapy due to the nontrivial rate of VTE events and the beneficial effects of anticoagulation on these events.

Patients with ISGDVT at risk of subsequent extension may benefit from anticoagulation, but identification of these patients is an unsolved question. Possible risk factors include: presence or traditional VTE risk factors (permanent more than transient), unprovoked DVT, D-Dimer, ISGDVT affecting more than $5 \mathrm{~cm}$ length or more than one vein, extent of ISGDVT at the second US, cancer, immobilization and hospitalization. None of these, or combination of these, have been formally tested for their predictive value. Hence, most of Physicians are left to rely on their own judgements.

In the present case, a risk factor for subsequent progression is the fact that DVT affects more than one vein (both soleal and gastrocnemius veins), in contrast, it seems to be a provoked event following a thigh hematoma and immobilization after metatarsal surgery, all of them known to be transient factors. No information is given regarding the length of the thrombus or the eventual progression in an eventual second CUS. Thus, given the lack of consensus regarding the benefits of anticoagulation therapy in patients with ISGDVT, I strongly believe that the key factor in our patient is the presence of an evolving thigh hematoma rather than the fact of presenting ISGDVT. In everyday clinical practice there is uncertainty about what to do in patients with ISGDVT, however it is compulsory in this particular case minimizing the bleeding risk and avoiding overtreatment.

A plausible strategy might be compression therapy combined with LMWH $40 \mathrm{mg}$ daily and follow-up CUS. As long as the patient presents active hemorrhage, administration of full dose anticoagulation therapy is not recommended. Aspirin should not be discontinued unless severe hemorrhage with clinical relevance such as hypotension and/or severe anemia are present.

In case of resolution of the thigh hematoma and progression of ISGDVT, a limited course of anticoagulation (short duration and/or reduced dosages with half therapeutic regimens of LMWH) combined with follow-up CUS to adjust treatment parameters according to thrombus progression could be considered.

\section{Conclusions}

Improvement in venous duplex technology have increased the detection of thrombi in soleal and gastrocnemius deep veins, which to date have been considered to be clinically insignificant. Many important issues, such as natural course, clinical relevance, risk of progression and optimal treatment of venous thrombosis limited to soleal and gastrocnemius veins still remain unclear which gives rise to discrepancies among Physicians. Hence, well-designed and properly powered clinical studies addressing the issue of ISGDVT need to be undertaken in order to assess the risks and benefits of anticoagulation in this setting.

\section{Acknowledgments}

We would like to thank the Thromboembolic Disease Chair of Universidad Católica de San Antonio de Murcia (UCAM) and Fundación para el Estudio de la Enfermedad Tromboembólica en España (FUENTE) for their initiative in the postgraduate course "Máster en Tratamiento Anticoagulante/Masters Degree in Anticoagulant Treatment", and S\&H Medical Science Service Training Center for its coordinating work.

\section{References}

1. Palareti G, Cosmi B, Lessiani G, Rodorigo G, Guazzaloca G, et al. (2010) Evolution of untreatedcalfdeep-veinthrombosis in high risk symptomatic outpatients: theblind, prospective CALTHRO study. Thromb Haemost 104: 1063-1070. [Crossref]

2. Kahn SR, Shrier I, Julian JA, Ducruet T, Arsenault L, et al. (2008) Determinants and time course of the post thrombotic syndrome after acute deep venoust hrombosis. Ann Intern Med 149: 698-707. [Crossref]

3. Masuda EM, Kistner RL, Musikasinthorn C, Liquido F, Geling O, et al. (2012) Thecontroversy of managing calfvein thrombosis. J Vasc Surg 55: 550-561. [Crossref]

4. Caggiati A, Bergan JJ, Gloviczki P, Eklof B, Allegra C, et al. (2005) Nomenclature of theveins of thelowerlimb: extensions, refinements, and clinicalapplication. $J$ Vasc Surg 41: 719-724. [Crossref]

5. Palareti G, Schellong S (2012) Isolated distal deepveinthrombosis: whatweknow and whatwe are doing. J Thromb Haemost 10: 11-19. [Crossref]

6. Galanaud JP, Quenet S, Rivron-Guillot K, Quere I, Sanchez Muñoz-Torrero JF, et al. (2009) Comparison of theclinicalhistory of symptomatic isolated distal deepveinthrombosis vs. proximal deepveinthrombosis in 11086 patients. J Thromb Haemost 7: 2028-2034. [Crossref]

7. Stein PD, Matta F, Musani MH, Diaczok B (2010) Silent pulmonary embolism in patients with deep venous thrombosis: a systematic review. Am J Med 123: 426-431. [Crossref]

8. Kahn SR, Shrier I, Julian JA, Ducruet T, Arsenault L, et al. (2008) Determinants and time course of the postthromboticsyndrome after acute deep venous thrombosis. Ann Intern Med 149: 698-707. [Crossref]

9. Goodacre S, Sampson F, Thomas S, van Beek E, Sutton A (2005) Systematicreview and meta-analysis of the diagnostic accuracy of ultrasonography for deep veinthrombosis. BMC MedImaging5: 6. [Crossref]

10. Schwarz T, Buschmann L, Beyer J, Halbritter K, Rastan A, et al. (2010) Therapy of isolated calfmus clevein thrombosis: a randomized, controlled study. J Vasc Surg 52 1246-1250. [Crossref]

11. Lautz TB, Abbas F, Walsh SJ, Chow C, Amaranto DJ, et al. (2010) Isolated gastrocnemius and solealveinthrombosis: should these patients receive therapeutic anticoagulation? Ann Surg 251: 735-742. [Crossref]

12. Macdonald PS, Kahn SR, Miller N, Obrand D (2003) Short-term natural history of isolatedgastrocnemius and solealveinthrombosis. J VascSurg37: 523-527. [Crossref]

13. Gillet JL, Perrin MR, Allaert FA (2007) Short-term and mid-termoutcome of isolated symptomatic muscular calfveinthrombosis. J Vasc Surg 46: 513-519. [Crossref]

14. Kearon C, Akl EA, Comerota AJ, Prandoni P, Bounameaux H, et al. (2012) American College of ChestPhysicians. Antithrombotic therapy for VTE disease: Antithrombotic Therapy and Prevention of Thrombosis, 9th ed: American College of Chest Physicians Evidence-Based Clinical Practice Guidelines. Chest 142: 1698-1704. [Crossref]

Copyright: (C2016 Gonzálvez AM. This is an open-access article distributed under the terms of the Creative Commons Attribution License, which permits unrestricted use, distribution, and reproduction in any medium, provided the original author and source are credited. 